\title{
ALMA-FORUM (ALMA-FACHEXPERTINNEN-FORUM)
}

\section{von Susanne Luger und Marian Miehl}

Zusammenfassung: In den Mitteilungen der VÖB 71(2) hat das Alma-FachexpertInnen-Forum über den Umstieg auf das neue Bibliotheksmanagementsystem Alma aus der Sicht der vormaligen Alma-Schulungsgruppe berichtet. In dieser Ausgabe präsentiert das Alma-Forum die aktuellen und zukünftigen Aufgaben, deren es sich gerne und mit Engagement annimmt.

Schlagwörter: Alma Fachexpertlnnen-Forum; Alma-Forum; Entwicklung; Mitglieder

\section{ALMA-FORUM (ALMA-FUNCTIONAL EXPERTS-FORUM)}

Abstract: In the Mitteilungen der VÖB 71(2) the Alma-Functional Experts-Forum reported their view as Alma-Schulungsgruppe on the implementation of the integrated library system Alma. In this issue the Alma-Forum presents the current and future assignments, which are coming to them.

Keywords: Alma-Functional Experts-Forum; Alma-Forum; development; members

DOI: https://doi.org/10.31263/voebm.v72i1.2281

(C) Susanne Luger, Marian Miehl

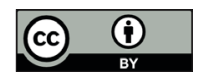

Dieses Werk ist lizenziert unter einer

Creative-Commons-Lizenz Namensnennung 4.0 International 
In einer der früheren Ausgaben der „Mitteilungen der VÖB“1 lag der Schwerpunkt auf den Erfahrungsberichten der Functional Experts Gruppen, die den Umstieg auf das neue Bibliothekssystem Alma vorbereiteten. Aus der Gruppe der Functional Experts entstand auch die Alma-Schulungsgruppe, deren Arbeitsauftrag mit dem Umstieg der Kohorte 2 offiziell beendet war.

Die Mitglieder der Schulungsgruppe wollten allerdings damit nicht aufhören, sondern auch für die bis 2022 nachkommenden Waves der restlichen Verbundbibliotheken ihr Wissen zur Verfügung stellen. Zu diesem Zweck wurde das Alma-FachexpertInnen-Forum geschaffen (kurz Alma-Forum), welches gleich einen personellen Zuwachs durch KollegInnen aus Bibliotheken der Wave 3 erfuhr. Das Alma-Forum versteht sich als offene Gruppe, der jederzeit beigetreten werden kann. Die Mitglieder decken die verschiedenen Bereiche von Alma ab und sind meist auch die Koordinatorlnnen der weiterhin stattfindenden WebCalls der einzelnen Fachbereiche.

Das Alma-Forum hält monatliche WebCalls ab, in denen verschiedene Punkte, etwa die Vorbereitung von Workshops für die einzelnen Waves, oder die Erarbeitung von unterstützenden Materialien, bzw. die Verwaltung der bisher erstellten Schulungsunterlagen, besprochen werden. Ebenso sind die monatlichen Release Notes ein Thema. Gemeinschaftlich werden die Neuerungen erarbeitet, damit die Erkenntnisse anschließend bei den WebCalls der einzelnen Gruppen weitergegeben werden können.

Das vordergründige Ziel des Alma-Forums ist daher im Moment die Aufrechterhaltung und Leitung der mit Beginn des Alma-Umstiegs eingerichteten Functional Experts Gruppen. Damit wird der von allen Beteiligten als positiv wahrgenommene Erfahrungsaustausch zwischen den einzelnen Bibliotheken gewährleistet und auftretende Probleme können so einfach kommuniziert und teilweise behoben werden.

Des Weiteren sieht sich das Alma-Forum als Knotenpunkt zwischen den einzelnen Fachbereichen und bspw. den Systembibliothekarlnnen. Durch den Austausch in den einzelnen Gruppen können Wünsche und Anregungen gesammelt werden, die in der Form oftmals die Systembibliothekarlnnen nicht erreichen. Dies spielt besonders in die Bereiche der verschiedenen Votings, die von Ex Libris eingerichtet worden sind, eine Rolle, da hier besondere Wünsche bzw. Funktionalitäten der Bearbeiterlnnen an Ex Libris herangetragen werden können. Um hier ein besseres Gewicht zu haben, wäre, in Abstimmung mit dem Fachbeirat, der Wunsch diese Abstimmungen österreichweit zu vereinheitlichen, damit 
die von uns eingebrachten Punkte eine höhere Chance bekommen auch umgesetzt zu werden.

In diesem Zusammenhang sieht sich das Alma-Forum auch als Knotenpunkt auf einer internationalen Ebene. Durch eine engere Zusammenarbeit mit den deutschen Alma-Anwenderbibliotheken, etwa über die DACHE$L A^{2}$, könnte den im deutschen Sprachraum vorherrschenden Besonderheiten mehr Gewicht für eine entsprechende Umsetzung durch Ex Libris gegeben werden.

Das Alma-Forum sieht sich demzufolge als Kommunikationsplattform, bei der verschiedene Stränge kanalisiert werden um die BibliotheksmitarbeiterInnen bei der Arbeit mit Alma zu unterstützen.

Die Leitlinien und weitere Informationen des Alma-Forums können auf der in Aufbau befindlichen Homepage nachgelesen werden.

Die aktuellen Mitglieder des Alma-Forums:

- Christian Beiler (UBW)

- Ingrid Bluch (UBK)

- Birgit Bittner (UBW)

- Victoria Buschbeck (AKW)

- Markus Heindl (BOKU)

- Werner Hirschmugl (VUW)

- Birgit Hörzer (UBG)

- Judith Lackner (TUW)

- Wolf-Dieter Lang (ONB)

- Susanne Luger (UBL) - Vorsitzende

- Martin Malina (VBK)

- Georg Mayr-Duffner (WUW)

- Marian Miehl (MUW) - stv. Vorsitzender

- J. Michael Mühlegger (UBS)

- Karl Rathmanner (FHSTP)

- Sandra Rindler (UBK)

- Verena Schaffner (OBVSG)

- Harald Schlinger (AKW)

- Sonja Schuh (UBG)

- Claudia Weitlaner (ULBT)

Im Folgenden finden Sie die einzelnen (Fach)ExpertInnengruppen mit den jeweiligen Ansprechpersonen und den Mailinglisten, über die der Erfahrungsaustausch stattfindet und Kontakt aufgenommen und bspw. die nächsten WebEx-Termine erfragt werden können. 
Alma FachexpertInnen-Forum

Sprecherlnnen: Susanne Luger (UBL), Marian Miehl (MUW)

Kontakt: alma-forum@obvsg.at

Fachexpertlnnengruppe Acquisitions

Ansprechpersonen: Sandra Rindler (UBK), Sonja Schuh (UBG)

Kontakt: alma-acq@obvsg.at

\section{ExpertInnengruppe E-Medien}

Ansprechpersonen: Birgit Bittner (UBW), Georg Mayr-Duffner (WUW)

Kontakt: eg-emedien@obvsg.at

FachexpertInnengruppe Fulfilment

Ansprechperson: Harald Schlinger (AKW)

Kontakt: alma-ful@obvsg.at

FachexpertInnengruppe Public Services

Ansprechperson: Nadine Cerny (OBVSG)

Kontakt: primo-info@obvsg.at

Fachexpertlnnengruppe Resource Management

Ansprechpersonen: Christian Beiler (UBW), Victoria Buschbeck (AKW)

Kontakt: alma-rm@obvsg.at

FachexpertInnengruppe Resource Sharing

Ansprechpersonen: Werner Hirschmugl (VUW), Sonja Klinger (UBS), Ingrid Tanzberger (ÖNB)

Kontakt: alma-rs@obvsg.at

FachexpertInnengruppe Zeitschriften

Ansprechperson: Claudia Weitlaner (UBI)

Kontakt:alma-zs@obvsg.at

Mag. . Susanne Luger Johannes Kepler Universität Linz, Universitätsbibliothek E-Mail: susanne.luger@jku.at

Mag. Marian Miehl, MSc Medizinische Universität Wien, Universitätsbibliothek E-Mail: marian.miehl@meduniwien.ac.at 
1 Bauer, Bruno; Lackner, Markus; Schubert, Bernhard: Implementierung des neuen Bibliotheksmanagementsystems Alma an 14 Einrichtungen im Österreichischen Bibliothekenverbund - Feedback aus der Perspektive der Functional Experts. In: Mitteilungen der VÖB 71 (2018) Nr. 2: ALMA im OBV, S. 320-350. https://doi.org/10.31263/voebm. v71i2.2136

2 DACHELA: http://dachela.org/ 